\title{
Contrast agents in Nephrology - a literature review
}

\author{
Sara Cardoso Fernandes ${ }^{1}$, Ana Carina Ferreira ${ }^{1,2}$ \\ 1 Department of Nephrology - Hospital Curry Cabral, Centro Hospitalar e Universitário de Lisboa Central \\ ${ }^{2}$ Nova Medical School | Faculdade de Ciências Médicas, Universidade Nova de Lisboa, Lisbon, Portugal
}

\section{ABSTRACT}

Contrast agents are widely used in ambulatory and hospitalized patients, as a complement to imaging studies, improving diagnostic accuracy. Patients with chronic kidney disease are at increased risk for adverse events related to contrast administration. In this review, we will summarize the current evidence on this topic.

Keywords: Radiocontrast, gadolinium, contrast-induced nephropathy, nephrogenic systemic fibrosis

\section{INTRODUCTION}

According to data from the Portuguese Registry of Dialysis and Transplantation 2018, the number of patients with chronic kidney disease (CKD) stage $5 \mathrm{~d}$ or $5 \mathrm{t}$ was 20,730 , meaning that the prevalence of patients on dialysis in Portugal is over 1.264 per million population ${ }^{1}$. We can conclude that the prevalence of CKD stage 3 to 5 is higher, given that the majority of CKD patients never reach dialysis. Nevertheless, a study published in 2011 revealed that, in 2008, the prevalence of CKD in Portugal was $6.1 \%^{2}$.

As others, CKD patients are often subjected to radiological examinations, some of which require contrast media, such as coronary angiography (since cardiovascular pathology is almost universally present in patients with $\mathrm{CKD}^{3}$ ), contrast-enhanced computer tomography (CT), magnetic resonance imaging (MRI) or even arteriovenous fistula angioplasty.

Although the risk of renal function deterioration associated with contrast administration is low in the general population ${ }^{4}$, it may be as high as $25 \%$ in patients with pre-existing renal impairment or with certain risk factors ${ }^{5,6}$. Moreover, the risk for complications associated with the use of contrast in MRI exams is also increased in patients with renal disease.

In this review, we aim to summarize risk factors that predispose to renal injury after exposure to contrast media, and the main preventive strategies studied so far. We will focus on contrast-enhanced CT and MRI enhanced with gadolinium-based contrasts.

\section{CONTRAST-ENHANCED CT AND CONTRAST- INDUCED NEPHROPATHY}

Contrast-induced nephropathy (CIN) is defined as an absolute or relative increase in serum creatinine that occurs after exposure to a contrast agent when other causes of renal impairment are excluded ${ }^{7}$. It is the third leading cause of acute kidney injury (AKI) in hospitalized patients, accounting for $11 \%$ of the cases ${ }^{8}$ and is associated with increased risk of mortality, cardiovascular events, renal failure, and prolonged hospitalization ${ }^{9}$. It has known risk factors and preventive strategies, as we will see.

\section{High osmolality vs. low osmolality and iso-osmolality contrast media}

Radiocontrast agents can be ionic or non-ionic and those are classified according to their osmolality. The early reports of contrastinduced nephropathy happened with ionic and high osmolality contrast media, which were proven to have a 3.3 times greater risk of causing nephropathy when compared to low osmolality contrast media ${ }^{10}$. Thus, hyperosmolal contrasts are not currently used. Nowadays, radiologists use ionic and non-ionic low-osmolal agents, which have higher osmolality than plasma, as well as non-ionic iso-osmolal agents, with a similar osmolality to plasma ${ }^{11}$.

It seems that the risk of developing CIN is allied to the osmolality and not to the charge by itself. Only one study compared ionic agents according to their osmolality, and the low-osmolal agents revealed to be less nephrotoxic than the hyperosmolal agents ${ }^{12}$. Most studies compared non-ionic low-osmolal agents with ionic hyperosmolal contrast media, and all concluded that low-osmolal agents are safer. Publications comparing ionic and non-ionic low-osmolal versus isoosmolal agents showed conflicting results(13-15) · However, two metaanalyses failed to demonstrate any difference between renal toxicity of those agents, except for one particular non-ionic low-osmolal agent (iohexol), that seemed to be more nephrotoxic ${ }^{15,16}$.

Thus, recent guidelines recommend the use of low osmolality and iso-osmolality agents, since they are associated with a lower risk of kidney injury. 


\section{Risk factors}

The clinical significance of CIN heightens the importance of identifying patients with highest risk of developing this condition. So far, procedure and patient-related risk factors have been studied.

Procedure-related factors include:

1. The use of high-osmolality agents, as explained before;

2. The high volume of contrast media;

3. The number of procedures and repeated administration within 72 hours after initial exposure ${ }^{17,18}$;

4. The local of administration, as it was also verified that intraarterial administration of contrast media is associated with higher risk of CIN when compared to the intravenous route ${ }^{19}$.

Patient-related factors include:

1. Pre-existing renal impairment, which is the strongest patientrelated risk factor (higher baseline serum creatinine levels is associated with higher risk) ${ }^{20,21}$

2. Older age, especially those above 75 years old;

3. Other co-morbidities, as advanced chronic heart failure and anemia. Diabetes mellitus is also commonly considered an important risk factor for CIN. However, a randomized, doubleblind multicenter trial performed more than 20 years ago showed that the incidence of $\mathrm{CIN}$ in diabetic patients with preserved renal function was low, proving that it is not an independent risk factor, in spite of contributing to $\mathrm{CIN}$ in patients with underlying CKD ${ }^{10}$. This means that among CKD patients, those with diabetes are at higher risk;

4. Volume depletion;

5. The use of nephrotoxic drugs, like non-steroidal anti-inflammatory drugs (NSAIDs), or even antibiotics $7,19,22$.

\section{Pathophysiological mechanisms}

Although not completely elucidated, the pathophysiological mechanisms by which contrast agents cause kidney injury comprise hemodynamic and indirect effects, as well as direct effects ${ }^{23,24}$.

Hemodynamic and indirect mechanisms include generation of reactive oxygen species (ROS) and vasoconstriction mediated by vasomotor molecules such as endothelin, nitric oxide and prostaglandins that reduce glomerular blood flow and medullary hypoxia. In addition, the increased blood viscosity induced by contrast media diminishes erythrocyte plasticity leading to microvascular thrombosis 24,25

Direct mechanisms are related with an intrinsic cytotoxic effect on tubular epithelial cells, leading to cell apoptosis and necrosis, which, in turn, contribute to tubular obstruction, increased intratubular pressure and decreased glomerular filtration rate (GFR). It is still a matter of debate if contrast agents lead to epithelial necrosis or just functional changes in those cells, as recovery from $\mathrm{CIN}$ is much faster than recovery from acute tubular necrosis from other causes.

\section{Preventive Strategies}

In order to avoid CIN, many preventive strategies have been proposed over the years, including intravenous volume expansion, pharmaceutical agents and renal replacement therapies. The first step is to identify patients at risk, and CKD patients should be the target population for active preventive measures.

Although there are still some contradictory findings regarding the benefit of some of those prophylactic measures, current strategies compromise peri-procedural intravenous crystalloid ${ }^{26,27}$ along with avoidance of nephrotoxic drugs and use of low or iso-osmolality contrast media in the lowest possible volume. According to American College of Radiology guidelines published in 2018, isotonic fluids are preferred and a protocol using intravenous isotonic saline at an infusion rate of $100 \mathrm{~mL}$ per hour for six to 12 hours before and four to 12 hours after the procedure is recommended ${ }^{11}$.

\section{Volume expansion}

Over the years, multiple trials have compared the preventive efficacy of intravenous isotonic sodium bicarbonate with isotonic sodium chloride, given the hypothesis that urine alkalization reduces generation of ROS and lessens oxidative stress, a mechanism involved in the pathogenesis of CIN. Though there are some conflicting results $^{28,29}$, a double-blind two-by-two factorial design trial by Weisbord et al. found no benefit of intravenous sodium bicarbonate over intravenous sodium chloride for the incidence of $\mathrm{CIN}$ or for the prevention of death, need for dialysis or persistent kidney impairment at 90 days ${ }^{30}$.

Despite current recommendations, a non-inferiority trial and its long-term follow-up (AMACING - A Maastricht Contrast-Induced Nephropathy Guideline) showed no significant difference between the incidence of $\mathrm{CIN}$ in the group receiving intravenous hydration and the group assigned to no prophylactic measures ${ }^{31,32}$. Nevertheless, this study had some limitations, including the small sample size, singlecenter, low rates of intra-arterial and interventional procedures, as well as, a small percentage of patients with severe CKD. Thus, it is impossible to conclude that volume expansion is ineffective based on these results.

\section{Pharmaceutical agents}

$\mathrm{N}$-acetylcysteine is an agent presumed to reduce the incidence of $\mathrm{CIN}$ due to its vasodilatory and antioxidant effects ${ }^{33}$. Multiple trials and meta-analyses failed to reach a consensus on the role of this drug in CIN prophylaxis ${ }^{34}$, but the PRESERVE (Prevention of Serious Adverse Events Following Angiography) trial showed no reduction of the primary 90-day composite end-point comprising death, need for dialysis or persistent impairment of renal function or in the incidence of $\mathrm{CIN}^{30}$. Hence, the routine administration of $\mathrm{N}$-acetylcysteine is not advised despite being widely used due to its low costs and rare side effects.

Some observational studies also suggested that statins could have a protective effect given their pleiotropic properties (antiinflammatory and anti-oxidant effects). Indeed, the PRATO-ACS (Protective Effect of Rosuvastatin and Antiplatelet Therapy on Contrast-Induced Acute Kidney Injury and Myocardial Damage in 
Patients with Acute Coronary Syndrome) study showed a significant lower incidence of $\mathrm{CIN}$ in patients treated with high-dose rosuvas$\operatorname{tatin}^{35}$. These findings, however, were not corroborated in the prospective trial PROMISS (Prevention of Radiocontrast MediumInduced Nephropathy Using Short-Term High-Dose Simvastatin in Patients with Renal Insufficiency Undergoing Coronary Angiography) ${ }^{36}$. Thus, further studies are needed in order to determine the role of statins in CIN prophylaxis.

Theophylline, a non-selective adenosine receptor antagonist, was thought to have a role in preventing $\mathrm{CIN}^{37}$; however, a meta-analysis performed in 2005 showed inconclusive results ${ }^{38}$.

Diuretics and forced diuresis were studied as a means of preventing $\mathrm{CIN}$, but failed to show any benefit. Both mannitol (an osmotic diuretic) and furosemide (a loop diuretic) were tested, due to their renal vasodilatory effects and promotion of tubular flow. However, these effects did not reduce the risk of $\mathrm{CIN}^{39,40}$.

\section{Renal replacement therapies}

Extracorporeal blood purification therapies were also studied for the prevention of CIN. A meta-analysis performed by Cruz et al. concluded that these treatments do not have significant effect on the incidence of $\mathrm{CIN}^{41,42}$, and thus it is not recommended to perform dialysis after contrast injection.

\section{Current Paradigm}

Despite all the previous reports published in the past decades that support the association of contrast media and renal function impairment, more recent studies have been questioning this evidence. In fact, the majority of these studies lack a control group of patients who is not exposed to intravenous contrast media. Moreover, they assume that any elevation of serum creatinine levels that meets the diagnostic criteria is a consequence of $\mathrm{CIN}$, not accounting for fluctuations in creatinine levels that have been recognized as a common phenomenon in hospitalized patients or other causes of $A \mathrm{Al}^{43,44}$. The work of Newhouse ${ }^{45}$ and Bruce ${ }^{46}$ showed that the incidence of creatinine elevation in subjects who underwent unenhanced CT was similar to those who received intravascular iodinated contrast agents. These findings suggest that the incidence of CIN may be overestimated. However, those studies may have a selection bias in which higher-risk patients are less likely to be exposed to contrast material.

A meta-analysis performed by McDonald et al. comprising 25,950 patients failed to show a significant difference in the incidence of AKI, dialysis and death between patients who were subjected to procedures with intravenous administration of contrast material and controls ${ }^{47}$. This new evidence was corroborated by propensity-score matched studies that aimed to eliminate biases caused by comorbidities and confounding factors ${ }^{48-51}$.

Although currently available evidence is insufficient to declare that contrast media are not nephrotoxic, patients should be carefully evaluated and assessed for comorbidities and factors that confer higher risk so that evidence-based preventive measures can be implemented.

\section{MAGNETIC RESONANCE IMAGING AND NEPHROGENIC SYSTEMIC FIBROSIS}

Given the widely known nephrotoxicity of radiocontrast agents, MRI scans enhanced with gadolinium-based contrast (GBC) agents have been considered a safe alternative to CT scans, when the need for contrast media is imperative. Meanwhile, concerns about gadolinium toxicity came to light and a new clinical entity was recognized as a consequence of exposure to this agent in CKD patients - nephrogenic systemic fibrosis (NSF) ${ }^{52}$.

NSF, originally termed nephrogenic fibrosing dermopathy, was first reported in $1997^{53}$. In 2000, Cowper et a ${ }^{54}$. described the case of nine renal transplant recipients who required long-term dialysis, five patients with ESRD, and one patient with AKI identified with a skin condition characterized by thickening and hardening of the skin of the extremities, histologically similar to scleromyxedema.

Evidence linking NSF to GBC agents exposure emerged a few years later ${ }^{55,56}$. Marckmann et al. presented a description of 13 patients with end-stage CKD, all of which had been exposed to gadodiamide before the development of NSF. The time period between exposure and development of the first symptoms of the disease varied between two and 75 days $^{56}$.

Further evidence made the association clear when High et al. demonstrated that affected tissue of patients with NSF is approximately 35- to 150-fold higher than the level of retained gadolinium in the bone of healthy controls 57 .

As a systemic disease, NSF not only is responsible for cutaneous hyperpigmentation and induration and joint contractures, but it also causes fibrosis of the left ventricular myocardium, pericardium, great vessels, interstitial pulmonary fibrosis and pulmonary hypertension. It has a significant burden in mortality, with a 24-month mortality rate of $48 \%$ versus $20 \%$ of controls $^{58}$.

This entity develops due to infiltration of affected tissues by macrophages and fibroblasts, which release profibrotic cytokines, as transforming growth factor- beta-1. The diagnosis is established with a skin biopsy showing fibrotic lesions, stained with CD34+ dermal dendritic cells.

\section{Gadolinium-based contrast}

GBC agents are nonradioactive, paramagnetic, non-tissue specific, hyperosmolal contrast agents, composed of gadolinium bound to a chelating ligand. They are classified according to their charge (ionic and non-ionic) and their chelating ligand (linear or macrocyclic $)^{11}$. The chelating ligand is crucial to the molecule's safety, since free gadolinium is toxic, as it can precipitate in tissues and reduce neuromuscular transmission by blocking calcium channels. Ionic and macrocyclic agents are more strongly bound and, thus, more stable ${ }^{59}$. These agents are mostly excreted by the kidney, since they don't bind to proteins, although two of them are also excreted in bile. 
They can be classified into three groups, according to their likelihood of causing NSF${ }^{38}$ :

- Group 1: molecules with a linear chelating agent and with a strong association with NSF. These agents are contraindicated in dialysis and acute renal injury patients and are not recommended in CKD patients with eGFR $<30 \mathrm{ml} / \mathrm{min}$;

- Group 2: most molecules with a macrocyclic chelating agent (except for one molecule - gadobenate), and a weak association with NSF;

- Group 3: newer agents, with insufficient data.

\section{Risk factors for NSF}

As with $\mathrm{CIN}$, procedure and patient-related risk factors have been studied.

Procedure-related factors include:

1. The use of $G B C$ agents from group 1, as explained before;

2. The use of GBC agents with linear chelating preparations;

3. The high volume and dose of the agent;

4. The intra-arterial route(60-63).

Patient-related factors include:

5. Pre-existing renal impairment. It is important to notice that patients at highest risk of developing NSF are those with underlying renal dysfunction. The majority of cases described occur in patients with end-stage CKD undergoing hemodialysis. However, there are some cases reported in patients with stage 4 and 3 CKD, as well as, patients with acute kidney injury or after renal transplant ${ }^{64}$;

6. Other potential risks, not proved, are high phosphate or iron serum levels, metabolic acidosis, high-dose erythropoietin and concomitant use of lanthanum carbonate ${ }^{65}$.

\section{Preventive strategies}

The main preventive strategy is avoiding Group I GBC agents or linear chelating GBC agents in patients with eGFR inferior to $30 \mathrm{~mL} / \mathrm{min}$.

In dialysis patients, if GBC agents are indispensable, only Group II or III agents should be used, and hemodialysis should be performed immediately after (within 1 to 4 hours). A second dialysis session within 24 hours should be considered ${ }^{66}$. In peritoneal dialysis (PD) patients, a hemodialysis catheter should be placed, and two sessions should be performed after the MRI. If this is not possible, PD must be performed using a regimen of 10 to 15 exchanges per day for two days, with no dry period ${ }^{67}$.

Further investigation is needed in order to find less nephrotoxic agents that can be an alternative to GBC agents. Ferumoxytol is a drug used as an iron replacement therapy in patients with chronic renal disease and, in spite of not being approved for use as an MRI contrast agent, has no known nephrotoxicity and may be considered when its clinical benefit outweighs the risk of allergic reaction ${ }^{68}$. Manganesebased complexes have also shown potential since they are cleared via biliary excretion, a particularly attractive feature in the context of renal compromise ${ }^{69}$.

\section{CONCLUSION}

CIN has been widely accepted as a clinical entity responsible for increased risk of mortality, cardiovascular events, renal failure, and prolonged hospitalization. In spite of the current controversy regarding its actual incidence, it is important to recognize this condition and prevent it. As the understanding of its pathophysiological mechanisms and risk factors has arisen, new evidence about preventive strategies has also emerged, making it imperative to thoroughly evaluate patients at highest risk of developing CIN and implement evidence-based prophylactic measures.

It is also important to be aware of GBC agents' toxicity and increased risk of NSF in advanced stages of CKD. Further investigation is needed to find less harmful agents that can constitute an alternative in MRI enhancement.

Also, less harmful imaging techniques should be preferred whenever possible and advances in the investigation are needed in order to find new contrast media without toxic potential. In every patient, risk-benefit ratio should be assessed so that the fear of CIN or NSF doesn't limit correct diagnosis and appropriate and timely management.

Disclosure of potential conflicts of interest: none declared

\section{References}

1. Galvão A, Filipe R, Carvalho MJ, et al. Portuguese Registry of Dialysis and Transplantation 2018. http://www.spnefro.pt: Sociedade Portuguesa de Nefrologia 2018

2. Vinhas J, Gardete-Correia L, Boavida JM et al. Prevalence of chronic kidney disease and associated risk factors, and risk of end-stage renal disease: data from the PREVADIAB study. Nephron Clin Pr 2011;119:35-40.

3. Cozzolino M, Mangano M, Stucchi A, et al. Cardiovascular disease in dialysis patients. Nephrol Dial Transpl 2018;33:28-34.

4. Berns A. Nephrotoxicity of contrast media. Kidney Int 1989;36(4):730-40.

5. Rudnick MR, Goldfarb S, Tumlin J. Contrast-Induced Nephropathy: Is the Picture any Clearer? Clin Am Soc Nephrol 2008;3(1):261-2

6. Khwaja A. KDIGO Clinical Practice Guideline for Acute Kidney Injury. Off J Int Soc Nephrol 2012;2(1):69-88.

7. Mehran R, Nikolsky E. Contrast-induced nephropathy : Definition, epidemiology, and patients at risk. Kidney Int 2006;69:S11-5.

8. Nash K, Hafeez A, Hou S. Hospital-acquired renal insufficiency. Am J Kidney Dis2002;39(5):930-6.

9. James MT, Samuel SM, Manning MA, et al. Contrast-induced acute kidney injury and risk of adverse clinical outcomes after coronary angiography: A systematic review and meta-analysis. Circ Cardiovasc Interv 2013;6(1):37-43.

10. Rudnick MR, Goldfarb S, Wexler L, et al. Nephrotoxicity of ionic and nonionic contrast media in 1196 patients: A randomized trial. Kidney Int 1995;47(1):254-61.

11. ACR Committee on Drugs and Contrast Media. ACR Manual on Contrast Media. American College of Radiology 2018. 1-128 p.

12. Lautin EM, Freeman NJ, Schoenfeld AH et al. Radiocontrast-associated renal dysfunction: a comparison of lower-osmolality and conventional high-osmolality contrast media. AJR Am J Roentgenol 1991;157:59-65.

13. Kuhn MJ, Chen N, Sahani DV, et al. The PREDICT study: A randomized double-blind comparison of contrast-induced nephropathy after low- or isoosmolar contrast agent exposure. Am J Roentgenol 2008;191(1):151-7.

14. McCullough PA, Bertrand ME, Brinker JA, et al. A Meta-Analysis of the Renal Safety of Isosmolar Iodixanol Compared With Low-Osmolar Contrast Media. J Am Coll Cardiol 2006;48(4):692-9.

15. Eng J, Wilson RF, Subramaniam RM, et al. Comparative effect of contrast media type on the incidence of contrast-induced nephropathy a systematic review and meta-analysis. Ann Intern Med 2016;164(6):417-24. 
16. Reed M, Meier P, Tamhane UU, et al. The Relative Renal Safety of lodixanol Compared With LowOsmolar Contrast Media. A Meta-Analysis of Randomized Controlled Trials. JACC Cardiovasc Interv 2009;2(7):645-54.

17. Mehran R, Aymong ED, Nikolsky E, et al. A simple risk score for prediction of contrast-induced nephropathy after percutaneous coronary intervention: Development and initial validation. J Am Coll Cardiol 2004;44(7):1393-9.

18. Maioli M, Toso A, Gallopin M, et al. Preprocedural score for risk of contrast-induced nephropathy in elective coronary angiography and intervention. J Cardiovasc Med 2010;11(6):444-9.

19. Toprak O. Conflicting and New Risk Factors for Contrast Induced Nephropathy 2007;178(6):227783.

20. Rihal CS, Textor SC, Grill DE, et al. Incidence and prognostic importance of acute renal failure after percutaneous coronary intervention. Circulation 2002;105(19):2259-64.

21. McCullough PA, Wolyn R, Rocher LL, et al. Acute Renal Failure After Coronary Intervention. Am J Med2002;103(5):368-75.

22. McCullough PA, Adam A, Becker CR, et al. Risk Prediction of Contrast-Induced Nephropathy. Am J Cardiol 2006;98:27-36.

23. Heyman SN, Clark BA, Kaiser N, et al. Radiocontrast agents induce endothelin release in vivo and in vitro. J Am Soc Nephrol 1992;3(1):58-65.

24. Persson PB, Hansell P, Liss P. Pathophysiology of contrast medium - induced nephropathy. Kidney Int 2005;68:14-22.

25. Seeliger E, Sendeski M, Rihal CS, et al. Contrast-induced kidney injury: Mechanisms, risk factors, and prevention. Eur Heart J 2012;33(16):2007-15.

26. Mueller C, Buerkle G, Buettner HJ, et al. Prevention of Contrast Media-Associated Nephropathy. Arch Intern Med 2003;162(3):329.

27. Trivedi HS, Moore H, Nasr S, et al. A Randomized Prospective Trial to Assess the Role of Saline Hydration on the Development of Contrast Nephrotoxicity. Nephron Clin Pract 2004;93(1):c29-34.

28. Shen AY, Jorgensen MB, Kotlewski A, et al. Sodium Bicarbonate vs Sodium Chloride for the Prevention of Contrast Undergoing Coronary Angiography 2015;300(9)

29. Ozcan EE, Guneri S, Akdeniz B, et al. Sodium bicarbonate, N-acetylcysteine, and saline for prevention of radiocontrast-induced nephropathy. A comparison of 3 regimens for protecting contrastinduced nephropathy in patients undergoing coronary procedures. A single-center prospective controlled. Am Heart J 2007;154(3):539-44.

30. Weisbord SD, Gallagher M, Jneid H, et al. Outcomes after Angiography with Sodium Bicarbonate and Acetylcysteine. N Engl J Med 2018;378(7):603-14.

31. Nijssen EC, Rennenberg RJ, Nelemans PJ, et al. Prophylactic hydration to protect renal function from intravascular iodinated contrast material in patients at high risk of contrast-induced nephropathy (AMACING): a prospective, randomised, phase 3, controlled, open-label, non-inferiority trial. Lancet 2017;389(10076):1312-22.

32. Nijssen EC, Nelemans PJ, Rennenberg RJ, et al. Prophylactic Intravenous Hydration to Protect Renal Function From Intravascular lodinated Contrast Material (AMACING): Long-term Results of a Prospective, Randomised, Controlled Trial. Lancet 2018;389 (10076):109-16.

33. Sterling KA, Tehrani T, Rudnick MR. Clinical significance and preventive strategies for contrastinduced nephropathy. Curr Opin Nephrol Hypertens 2008;17(6):616-23.

34. Kelly AM, Dwamena B, Cronin P,et al. Meta-analysis: effectiveness of drugs for preventing contrastinduced nephropathy. Ann Intern Med 2008;148(4):284-94.

35. Leoncini M, Toso A, Maioli M, et al. Early high-dose rosuvastatin for contrast-induced nephropathy prevention in acute coronary syndrome: Results from the PRATO-ACS study (protective effect of rosuvastatin and antiplatelet therapy on contrast-induced acute kidney injury and myocardia damage. J Am Coll Cardiol 2014;63(1):71-9.

36. Jo SH, Koo BK, Park JS, et al. Prevention of radiocontrast medium-induced nephropathy using short-term high-dose simvastatin in patients with renal insufficiency undergoing coronary angiography (PROMISS) trial-a randomized controlled study. Am Heart J2008;155(3):1-8.

37. Kapoor A. The role of theophylline in contrast-induced nephropathy: a case-control study. Nephrol Dial Transplant 2002;17(11):1936-41.

38. Bagshaw SM. Theophylline for Prevention of Contrast-Induced Nephropathy. Arch Intern Med 2005;165(10):1087.

39. Majumdar SR, Kjellstrand CM, Tymchak WJ,et al. Forced Euvolemic Diuresis With Mannitol and Furosemide for Prevention of Contrast-Induced Nephropathy in Patients With CKD Undergoing Coronary Angiography: A Randomized Controlled Trial. Am J Kidney Dis 2009;54(4):602-9.

40. Solomon R, Werner C, Mann D, et al. Effects of Saline, Mannitol and Furosemide on Acute Decreases in Renal Function Induced by Radiocontrast Agents. N Engl J Med 1994;331:1416-20.

41. Cruz DN, Perazella MA, Bellomo R, et al. Extracorporeal Blood Purification Therapies for Prevention of Radiocontrast-Induced Nephropathy: A Systematic Review. Am J Kidney Dis 2006;48(3):361-71.

42. Cruz DN, Goh CY, Marenzi G, et al. Renal replacement Therapies for prevention of radiocontrastinduced nephropathy: A systematic review. Am J Med2012;125(1):66-78.

43. Davenport MS, Dillman JR, Cohan RH, et al. Contrast Material - induced Nephrotoxicity and Intravenous 2013;267(1):94-105
44. Gallardo X, Almirall J, Castaner E, et al. Assessing the nephrotoxicity of intravenous iodinated contrast agents in patients with chronic renal failure in the absence of interfering acute disease. Acta radiol 2019;1-5.

45. Newhouse JH, Kho D, Rao QA, et al. Frequency of serum creatinine changes in the absence of iodinated contrast material: Implications for studies of contrast nephrotoxicity. Am J Roentgenol 2008;191(2):376-82.

46. Bruce RJ, Djamali A, Shinki K, et al. Background fluctuation of kidney function versus contrastinduced nephrotoxicity. Am J Roentgenol 2009;192(3):711-8.

47. McDonald JS, McDonald RJ, Comin J, et al. Frequency of acute kidney injury following intravenous contrast medium administration: a systematic review and meta-analysis. Radiology 2013;267(1):119-28.

48. Mcdonald JS, Mcdonald RJ, Carter RE, et al. Risk of Intravenous Contrast Material - mediated Acute Kidney Injury : A Propensity Score - matched Study Stratified by Baseline-estimated Glomerular Filtration Rate. Radiology 2014;271(1):65-73.

49. Mcdonald RJ, Mcdonald JS, Bida JP, et al. Material - induced Nephropathy : Causal or Coincident Phenomenon ? Radiology 2013:267(1):106-19.

50. Mcdonald RJ, Mcdonald JS, Carter RE, et al. Intravenous Contrast Material Exposure Is Not an Independent Risk Factor for Dialysis or Mortality. Radiology 2014;273(3):714-725.

51. Mcdonald JS, Mcdonald RJ, Lieske JC, et al. Risk of Acute Kidney Injury, Dialysis and Mortality in Chronic Kidney Disease Patients following Intravenous Contrast Materal Exposure. Mayo Clin Proc 2015;90(8):1046-53.

52. Perazella MA. Current status of gadolinium toxicity in patients with kidney disease. Clin J Am Soc Nephrol 2009;4(2):461-9.

53. Centers for Disease Control and Prevention. Fibrosing skin condition among patients with renal disease - United States and Europe 1997-2002. MMWR Morb Mortal Wkly Rep 2002;51:25-6.

54. Cowper SE, Robin HS, Steinberg SM. Scleromyxoedema-like cutaneous diseases in renal-dialysis patients. Lancet 2000;356(2):1000-1.

55. Grobner T. Gadolinium - a specific trigger for the development of nephrogenic fibrosing dermopathy and nephrogenic systemic fibrosis? Nephrol Dial Transplant 2006;21(4):1104-8.

56. Marckmann P, Skov L, Rossen K, et al. Nephrogenic Systemic Fibrosis: Suspected Causative Role of Gadodiamide Used for Contrast-Enhanced Magnetic Resonance Imaging. J Am Soc Nephrol 2006;17(9):2359-62.

57. High WA, Eng M, Ayers RA, et al. Gadolinium is quantifiable within the tissue of patients with nephrogenic systemic fibrosis. J Am Acad Dermatol 2007;56(4):709-10.

58. Kay J. Nephrogenic systemic fibrosis : a gadolinium- associated fibrosing disorder in patients with renal dysfunction. Ann Rheum Dis 2008;67(1):66-70.

59. Perazella MA, Rodby RA. Gadolinium use in patients with kidney disease: A cause for concern. Semin Dial 2007;20(3):179-85

60. Erley CM, Bader BD, Berger ED, et al. Gadolinium-based contrast media compared with iodinated media for digital subtraction angiography in azotaemic patients. Nephrol Dial Transplant 2004;19(10):2526-31.

61. Ergün I, Keven K, Uruç I, et al. The safety of gadolinium in patients with stage 3 and 4 renal failure. Nephrol Dial Transplant 2006;21(3):697-700.

62. Briguori C, Colombo A, Airoldi F, et al. Gadolinium-based contrast agents and nephrotoxicity in patients undergoing coronary artery procedures. Catheter Cardiovasc Interv 2006;67(2):175-80.

63. Kane GC, Stanson AW, Kalnicka D, et al. Comparison between gadolinium and iodine contrast for percutaneous intervention in atherosclerotic renal artery stenosis: Clinical outcomes. Nephrol Dial Transplant 2008;23(4):1233-40.

64. Cassis TB, Jackson JM, Sonnier GB, et al. Nephrogenic fibrosing dermopathy in a patient with acute renal failure never requiring dialysis. Int J Dermatol 2006;45(1):56-9.

65. Abu-Alfa AK. Nephrogenic Systemic Fibrosis and Gadolinium-Based Contrast Agents. Adv Chronic Kidney Dis 2011;18(3):188-98.

66. Saitoh T, Hayasaka K, Tanaka Y, et al. Dialyzability of gadodiamide in hemodialysis patients. Radiat Med 2006;24:445-51.

67. Murashima M, Drott HR, Carlow D, et al. Removal of gadolinium by peritoneal dialysis. Clin Nephrol 2008;69:368-72.

68. Gale EM, Caravan P. Gadolinium-Free Contrast Agents for Magnetic Resonance Imaging of the Central Nervous System. ACS Chem Neurosci 2018;9(3):395-7.

69. Gale EM, Atanasova IP, Blasi F, et al. A Manganese Alternative to Gadolinium for MRI Contrast. J Am Chem Soc 2015;137(49):15548-57.

\section{Correspondence to:}

Ana Carina Ferreira, MD

Rua da Beneficência, n.요, 1069-166, Lisboa, Portugal

E-mail: a.carina.costa.ferreira@gmail.com 